\title{
ON WEAK STATIONARITY AND WEAK ISOTROPY OF PROCESSES OF CONVEX BODIES AND CYLINDERS
}

\author{
LARS MICHAEL HOFFMANN ${ }^{*}$ Universität Karlsruhe (TH)
}

\begin{abstract}
Generalized local mean normal measures $\mu_{z}, z \in \mathbb{R}^{d}$, are introduced for a nonstationary process $X$ of convex particles. For processes with strictly convex particles it is then shown that $X$ is weakly stationary and weakly isotropic if and only if $\mu_{z}$ is rotation invariant for all $z \in \mathbb{R}^{d}$. The paper is concluded by extending this result to processes of cylinders, generalizing Theorem 1 of Schneider (2003).
\end{abstract}

Keywords: Particle process; cylinder process; Poisson process; mean normal measure; weak stationarity; weak isotropy

2000 Mathematics Subject Classification: Primary 60D05

Secondary 52A22; 60G55

\section{Introduction}

For stationary processes of convex particles Weil [9], [10] introduced mean normal measures as measure-valued parameters. They can be used to define associated convex bodies and thus allow the application of convex geometric tools to analyze properties of the underlying process - an idea which goes back to Matheron [4], who studied stationary processes of hyperplanes via their associated zonoids. A detailed account of the stationary case can be found in [8, Section 4.5] (also note the references given in [8, p. 178ff]). Recently, Schneider [6] considered local direction measures for nonstationary processes of flats and proved that a flat process is weakly stationary and weakly isotropic if and only if its local direction measures are rotation invariant. In the present paper we prove a similar result for processes with strictly convex particles and a generalization for cylinder processes which includes and extends both special cases.

In Sections 2 and 3 we introduce some basic notation and a generalization of local mean normal measures (see [2]). Section 4 contains the result that processes with strictly convex particles are stationary and isotropic if and only if all their generalized local mean normal measures are rotation invariant. Finally, in Section 5 a more general result for cylinder processes is presented that includes both the main theorem from Section 4 and Theorem 1 of [6]. The appendices collect some well-known facts about convex particles and some auxiliary results which are needed throughout the paper.

\section{Preliminaries and basic notation}

Let $d \in \mathbb{N} \backslash\{0\}$. Throughout this paper we will work in $d$-dimensional Euclidean space $\mathbb{R}^{d}$, with $B^{d}$ being the unit ball, $S^{d-1}$ the unit sphere with spherical Lebesgue measure $\omega_{d-1}$, and

Received 13 December 2006; revision received 12 June 2007.

* Postal address: Institut für Algebra und Geometrie, Universität Karlsruhe (TH), 76128 Karlsruhe, Germany. Email address: lars.hoffmann@math.uni-karlsruhe.de 
$\lambda_{d}$ the $d$-dimensional Lebesgue measure. For a topological space $S$, we will denote the Borel $\sigma$-algebra by $\mathscr{B}(S)$ and the support of any Borel measure $v$ on $S$ by supp $v$. Furthermore, for any probability measure $\mathrm{P}$, let $\mathrm{E}_{\mathrm{P}}$ denote the expected value with respect to $\mathrm{P}$.

For $k \in\{0, \ldots, d\}$, the $k$-dimensional Hausdorff measure will be denoted by $\mathscr{H}^{k}$. Furthermore, let $\mathcal{L}_{k}^{d}$ and $\mathcal{E}_{k}^{d}$ be the Grassmannians of $k$-dimensional linear and affine subspaces of $\mathbb{R}^{d}$, respectively. In addition, let $S O_{d}$ and $G_{d}$ denote the spaces of all rotations and all rigid motions of $\mathbb{R}^{d}$, respectively. We denote by $L^{\perp}$ the orthogonal complement of a linear subspace $L$ and we denote by $\lambda_{L}$ the Lebesgue measure on $L$. By id we denote the identity map.

The space $\widetilde{F}^{\prime}$ of all nonempty closed subsets of $\mathbb{R}^{d}$ shall, as usual, be endowed with the Fell topology and the $\sigma$-algebra $\mathscr{B}\left(\mathcal{F}^{\prime}\right)$. The subspace $\mathcal{K}^{\prime} \subseteq \mathcal{F}^{\prime}$ of all nonempty compact convex sets, i.e. convex bodies, and the subset $\mathcal{K}_{0} \subseteq \mathcal{K}^{\prime}$ of all convex bodies with Steiner point (see Appendix A) in the origin shall be equipped with the Hausdorff metric $d_{\mathrm{H}}$ and the respective Borel $\sigma$-algebras. A particle process is called (weakly) stationary if its distribution (intensity measure) is invariant under translations and (weakly) isotropic if its distribution (intensity measure) is invariant under rotations. All basic concepts from stochastic geometry (e.g. point processes, intensity measures, or Campbell's theorem) can be found in [8].

For any $K \in \mathcal{K}^{\prime}$, we denote its affine hull, interior, boundary, and relative boundary by aff $K$, int $K$, bd $K$, and rel bd $K$, respectively. As usual, the dimension $\operatorname{dim} K$ of a convex body $K$ is defined as the dimension of its affine hull. Furthermore, $K$ is called strictly convex if its boundary does not contain any segment. If $K$ is a $d$-dimensional convex body and $x \in \operatorname{bd} K$, a regular boundary point of $K$, by definition there exists a unique outer unit normal vector of $K$ at $x$; let this vector be denoted by $\sigma_{K}(x)$ and let reg $K \subseteq$ bd $K$ be the set of all regular boundary points of $K$. Analogously, if $K$ is a lower-dimensional convex body and $x$ is a point on the relative boundary of $K$ with unique outer unit normal vector in aff $K$, let the latter be denoted by $\tilde{\sigma}_{K}(x)$. Finally, let $\Xi_{d-1}(K, \cdot)$ and $C_{d-1}(K, \cdot)=\Xi_{d-1}\left(K, \cdot \times S^{d-1}\right)$ respectively denote the $(d-1)$ th support measure and the $(d-1)$ th curvature measure of $K$. If $\operatorname{dim} K=d$, for $B \in \mathscr{B}\left(\mathbb{R}^{d}\right)$ and $A \in \mathscr{B}\left(S^{d-1}\right)$, we have $\Xi_{d-1}(K, B \times A)=\mathscr{H}^{d-1}\left(\mathrm{bd} K \cap B \cap \sigma_{K}^{-1}(A)\right)$, i.e. $\Xi_{d-1}(K, B \times A)$ is equal to the $(d-1)$-dimensional Hausdorff measure of all regular boundary points of $K$ that lie in $B$ and have outer unit normal vector in $A$. For all basic notions from convex geometry, we refer the reader to [5]. Some definitions and results important for this paper are also given in Appendix A.

In Sections 3 and 4 the measurability of the mappings we use follows from respective theorems in [5], [7], and [8], and Lemma B.2. In Section 5 the measurability is implied by an auxiliary result that we prove in Appendix B.

\section{Generalized local mean normal measures}

In the following two sections $X$ will always be a particle process on $\mathcal{K}^{\prime}$ with nontrivial locally finite intensity measure $\Theta$. Furthermore, we assume that $\Theta$ is of the form

$$
\Theta(\mathcal{A})=\int_{\mathcal{K}_{0}} \int_{\mathbb{R}^{d}} \mathbf{1}_{\mathcal{A}}(K+x) f(x) \lambda_{d}(\mathrm{~d} x) \mathrm{P}_{0}(\mathrm{~d} K), \quad \mathcal{A} \in \mathscr{B}\left(\mathcal{K}^{\prime}\right) .
$$

Here, $f: \mathbb{R}^{d} \rightarrow[0, \infty)$ denotes a continuous function and $\mathrm{P}_{0}$ denotes a probability measure on $\mathcal{K}_{0}$. Note that both $f$ and $\mathrm{P}_{0}$ are uniquely determined by $\Theta$; see [1, p. 173]). As usual, we call $f$ the intensity function of $X$. The mapping

$$
\begin{aligned}
\Phi: \mathcal{K}^{\prime} & \rightarrow \mathbb{R}^{d} \times \mathcal{K}_{0}, \\
K & \mapsto(K-s(K), s(K)),
\end{aligned}
$$


is a homeomorphism; see [8, p. 121]. Thus, $\Phi(X)$ is a point process on $\mathbb{R}^{d} \times \mathcal{K}_{0}$, a so-called 'germ-grain-process' (see [8, Section 4.3]), and the distribution of the typical grain is given by $\mathrm{P}_{0}$. Therefore, any random closed set $Z$ with distribution $\mathrm{P}_{0}$ will be called the typical particle of $X$ and we will refer to $\mathrm{P}_{0}$ as the distribution of the typical particle of $X$. If the latter is rotation invariant we call the typical particle of $X$ isotropic.

Let $z \in \mathbb{R}^{d}, A \in \mathcal{B}\left(S^{d-1}\right)$, and $\mathcal{A} \in \mathcal{B}\left(\mathcal{K}_{0}\right)$. We define a set function $\mu_{z}: \mathcal{B}\left(S^{d-1}\right) \times$ $\mathscr{B}\left(\mathcal{K}_{0}\right) \rightarrow[0, \infty)$ by

$$
\mu_{z}(A, \mathcal{A}):=\mathrm{E}_{\mathrm{P}_{0}} \mathbf{1}_{\mathcal{A}}(K) \int_{\mathbb{R}^{d}} f(z-x) \Xi_{d-1}(K, \mathrm{~d} x \times A) .
$$

Let $A \in \mathcal{B}\left(S^{d-1}\right)$ and $B \in \mathcal{B}\left(\mathbb{R}^{d}\right)$ be bounded. In the proof of Theorem 5.1, given in Section 5, it is shown that

$$
\mathrm{E} \sum_{K \in X} \mathscr{H}^{d-1}\left(B \cap \sigma_{K}^{-1}(A)\right)=\int_{\mathbb{R}^{d}} \mathbf{1}_{B}(z) \mu_{z}\left(A, \mathcal{K}_{0}\right) \lambda_{d}(\mathrm{~d} z) .
$$

As a Borel measure on $S^{d-1}$, the left-hand side can (after normalization) be interpreted as the distribution of the outer unit normal vectors of the particles of $X$ at boundary points in $B$; see [8, p. 157]. Letting $B$ shrink to $z$ yields a normalization of $\mu_{z}\left(\cdot, \mathcal{K}_{0}\right)$ that can be interpreted as the distribution of the outer unit normal vectors of the particles of $X$ at $z$ whenever $\mu_{z}\left(S^{d-1}, \mathcal{K}_{0}\right)>$ 0 . For this reason, $\mu_{z}\left(\cdot, \mathcal{K}_{0}\right)$ was called the local mean normal measure of $X$ at $z$ in [2]. Now let $\mathcal{A} \in \mathscr{B}\left(\mathcal{K}_{0}\right)$ such that $\mathrm{P}_{0}(\mathcal{A})>0$. Then $X_{\mathcal{A}}:=\Phi^{-1}(\{(x, K) \in \Phi(X) \mid K \in \mathcal{A}\})$ is a nonstationary process of convex particles and its local mean normal measure is (up to a constant) given by $\mu_{z}(\cdot, \mathcal{A})$. Therefore, we call $\mu_{z}$ the generalized local mean normal measure of $X$ at $z$. Let us additionally assume that $X$ is Poisson and let $Z_{\mathcal{A}}$ be the Boolean model generated by $X_{\mathcal{A}}$. In this case $\mu_{z}$ also has another interpretation. For any $z \in \mathbb{R}^{d}$, the volume density $\bar{p}(z)$ of $Z_{\mathcal{A}}$ at $z$ is defined as the probability that $z \in Z_{\mathcal{A}}$. Furthermore, let $\Xi_{d-1}^{+}$denote the extension of $\Xi_{d-1}$ to the extended convex ring; see [3, Section 3] for details. From Theorem 4.11 of [3] we find that, for arbitrary $A \in \mathcal{B}\left(S^{d-1}\right)$, the Borel measure

$$
\mathrm{E} \Xi_{d-1}^{+}\left(Z_{\mathcal{A}}, \cdot \times A\right)
$$

on $\mathbb{R}^{d}$ is absolutely continuous with respect to $\lambda_{d}$ with density

$$
z \mapsto \frac{1-\bar{p}(z)}{\mathrm{P}_{0}(\mathcal{A})} \mu_{z}(A, \mathcal{A}) \text {. }
$$

Hence, if $\mu_{z}\left(S^{d-1}, \mathcal{A}\right)>0$ a normalization of $\mu_{z}(\cdot, \mathcal{A})$ can be interpreted as the distribution of the normal vectors of $Z_{\mathcal{A}}$ at $z$.

Some important properties of $\mu_{z}$ are summarized in the following theorem.

Theorem 3.1. Let $X$ be a particle process with intensity measure as in (3.1), let $z \in \mathbb{R}^{d}$, and let $\mu_{z}$ be as given above. Then the set function

$$
\mu_{z}: \mathscr{B}\left(S^{d-1}\right) \times \mathscr{B}\left(\mathcal{K}_{0}\right) \rightarrow[0, \infty),(A, \mathcal{A}) \mapsto \mu_{z}(A, \mathcal{A}),
$$

can be extended to a measure on $\mathscr{B}\left(S^{d-1}\right) \otimes \mathscr{B}\left(\mathcal{K}_{0}\right)$. It is uniquely determined by $X$ and, for $\lambda_{d}$-almost all $z \in \mathbb{R}^{d}$, it is finite.

Proof. The assertions follow immediately from Theorem 5.1, below.

Note that $\mu_{z}(\cdot, \mathcal{A})$ is not necessarily an even measure. 


\section{A characterization of weak stationarity and weak isotropy}

Let $X$ be a particle process with intensity measure as in (3.1). In addition, assume that some $K \in \operatorname{supp} \mathrm{P}_{0}$ are strictly convex. Our main result in this section shows that in this case $X$ is weakly stationary and weakly isotropic if and only if the generalized local mean normal measure $\mu_{z}$ is rotation invariant for every $z \in \mathbb{R}^{d}$. This transfers a similar result by Schneider for processes of $k$-flats (see [6, Theorem 1]) to processes of convex particles. However, we start by characterizing isotropy of the typical particle of $X$ and weak stationarity of $X$, respectively, by invariance properties of $\mu_{z}$. Both proofs contain ideas that are vital for the derivation of the main theorem.

Let $X$ be a particle process with intensity measure as in (3.1). We say that $X$ satisfies condition $(A)$ if a typical particle of $X$ is almost surely $(d-1)$ - or $d$-dimensional and we say that $X$ satisfies condition $(B)$ if there exists a strictly convex $K_{0} \in \mathcal{K}_{0}$ such that, for all $\varepsilon>0$, we have

$$
\mathrm{P}_{0}\left(\left\{K \in \mathcal{K}_{0} \mid d_{\mathrm{H}}\left(K, K_{0}\right)<\varepsilon\right\}\right)>0,
$$

i.e. there exists a strictly convex $K_{0} \in \operatorname{supp} \mathrm{P}_{0}$.

Example. Let $d=2$. For $n \in \mathbb{N}$, let $R_{2 n}$ denote the regular polytope with $2 n+2$ vertices, all of which are assumed to be lying on the unit circle. Let $X$ be a particle process with intensity measure as in (3.1) and typical particle distribution $\mathrm{P}_{0}$ given by

$$
\mathrm{P}_{0}=\sum_{n=1}^{\infty} \frac{1}{2^{n}} \delta_{R_{2 n}}
$$

Here, for $K \in \mathcal{K}_{0}, \delta_{K}$ denotes the Dirac measure concentrated on $K$. Then $X$ satisfies condition (B) with $K_{0}=B^{2}$ even though its typical particle is almost surely a polytope. Hence, condition (B) does not impose any regularity properties on the typical particle of $X$. Obviously, similar examples can be given for the case in which $d>2$.

For any rotation $\vartheta \in S O_{d}$, let $\vartheta$ also denote the mapping $K \mapsto \vartheta K$ from $\mathcal{K}^{\prime}$ to $\mathcal{K}^{\prime}$.

Theorem 4.1. Let $X$ be a particle process with intensity measure as in (3.1). Furthermore, we assume that $X$ satisfies condition (A). Then the typical particle of $X$ is isotropic if and only if

$$
\mu_{z}\left(S^{d-1}, \mathcal{A}\right)=\mathrm{E}_{\vartheta \circ \mathrm{P}_{0}} \mathbf{1}_{\mathcal{A}}(K) \int_{\mathbb{R}^{d}} f(z-x) C_{d-1}(K, \mathrm{~d} x)
$$

for all $z \in \mathbb{R}^{d}, \mathcal{A} \in \mathcal{B}\left(\mathcal{K}_{0}\right)$, and $\vartheta \in S O_{d}$.

Proof. For $d=1$, the theorem is trivial; thus, let $d \geq 2$. Obviously, (4.1) holds if $\mathrm{P}_{0}$ is rotation invariant. Therefore, we assume that (4.1) holds. For all $z \in \mathbb{R}^{d}, \mathcal{A} \in \mathcal{B}\left(\mathcal{K}_{0}\right)$, and $\vartheta \in S O_{d}$ we then have

$$
\begin{aligned}
\mu_{z}\left(S^{d-1}, \mathcal{A}\right) & =\mathrm{E}_{\mathrm{P}_{0}} \mathbf{1}_{\mathcal{A}}(K) \int_{\mathbb{R}^{d}} f(z-x) C_{d-1}(K, \mathrm{~d} x) \\
& =\mathrm{E}_{\vartheta \circ \mathrm{P}_{0}} \mathbf{1}_{\mathcal{A}}(K) \int_{\mathbb{R}^{d}} f(z-x) C_{d-1}(K, \mathrm{~d} x) \quad(\text { by }(4.1)) \\
& =\mathrm{E}_{\vartheta} \circ \mathrm{P}_{0} \mathbf{1}_{\mathcal{A}}(K) \int_{\mathbb{R}^{d}} f(z-\vartheta x) C_{d-1}\left(\vartheta^{-1} K, \mathrm{~d} x\right) \\
& =\mathrm{E}_{\mathrm{P}_{0}} \mathbf{1}_{\vartheta-1}(K) \int_{\mathbb{R}^{d}} f(z-\vartheta x) C_{d-1}(K, \mathrm{~d} x) .
\end{aligned}
$$


For the third equality we used the rotation covariance of $C_{d-1}$. For all $\vartheta \in S O_{d}$ and $\mathcal{A} \in \mathscr{B}\left(\mathcal{K}_{0}\right)$, this implies that $\mathrm{P}_{0}(\mathcal{A})=0$ if and only if $\mathrm{P}_{0}(\vartheta \mathscr{A})=0$. Otherwise, without loss of generality, there would exist a set $\mathcal{A} \in \mathscr{B}\left(\mathcal{K}_{0}\right)$ with $\mathrm{P}_{0}(\mathcal{A})>0$ and $\mathrm{P}_{0}(\vartheta \mathcal{A})=$ 0 . Let $\mathcal{K}_{0}^{(n)}:=\left\{K \in \mathcal{K}_{0} \mid \operatorname{dim} K \in\{d-1, d\}, K \subseteq n B^{d}\right\}$. We choose $n \in \mathbb{N}$ such that $\mathrm{P}_{0}\left(\mathcal{A} \cap \mathcal{K}_{0}^{(n)}\right)>0$. Using Lemma A.2 it is then easy to obtain a contradiction to (4.1). Hence, for all $\vartheta \in S O_{d}$, there exists a measurable function $\eta_{\vartheta}: \mathcal{K}_{0} \rightarrow[0, \infty)$ such that

$$
\mathrm{E}_{\mathrm{P}_{0}} g(K)=\mathrm{E}_{\vartheta} \circ \mathrm{P}_{0} g(K) \eta_{\vartheta}(K)=\mathrm{E}_{\mathrm{P}_{0}} g(\vartheta K) \eta_{\vartheta}(\vartheta K)
$$

for any measurable mapping $g: \mathcal{K} \rightarrow[0, \infty)$. Let there exist an $r(n) \geq 0, n \in \mathbb{N}$, as in Lemma A.2, i.e. such that

$$
\int_{\mathbb{R}^{d}} \int_{r(n) B^{d}} f(z-\vartheta x) \lambda_{d}(\mathrm{~d} z) C_{d-1}(K, \mathrm{~d} x)>0
$$

for all $K \in \mathcal{K}_{0}^{(n)}$. Repeating the calculation from the beginning of the proof, we obtain, from (4.1) and (4.2),

$$
\begin{aligned}
\mathrm{E}_{\mathrm{P}_{0}} \mathbf{1}_{\vartheta} \vartheta_{\mathcal{A}}(K) \int_{\mathbb{R}^{d}} \int_{r(n) B^{d}} f(z-\vartheta x) \lambda_{d}(\mathrm{~d} z) C_{d-1}(K, \mathrm{~d} x) \\
\quad=\mathrm{E}_{\mathrm{P}_{0}} \mathbf{1}_{\vartheta^{-1} \mathcal{A}}(K) \int_{\mathbb{R}^{d}} \int_{r(n) B^{d}} f(z-\vartheta x) \lambda_{d}(\mathrm{~d} z) C_{d-1}(K, \mathrm{~d} x) \eta_{\vartheta}(\vartheta K)
\end{aligned}
$$

for all $\mathcal{A} \in \mathscr{B}\left(\mathcal{K}_{0}\right)$. Thus, for $\mathrm{P}_{0}$-almost all $K \in \mathcal{K}_{0}^{(n)}$, we have

$$
\begin{aligned}
\int_{\mathbb{R}^{d}} & \int_{r(n) B^{d}} f(z-\vartheta x) \lambda_{d}(\mathrm{~d} z) C_{d-1}(K, \mathrm{~d} x) \\
& =\int_{\mathbb{R}^{d}} \int_{r(n) B^{d}} f(z-\vartheta x) \lambda_{d}(\mathrm{~d} z) C_{d-1}(K, \mathrm{~d} x) \eta_{\vartheta}(\vartheta K),
\end{aligned}
$$

which implies that $\eta_{\vartheta}(\vartheta K)=1$. Letting $n$ tend to infinity we obtain $\eta_{\vartheta}(\vartheta K)=1$ for $\mathrm{P}_{0}$-almost all $K \in \mathcal{K}_{0}$. Hence, $\mathrm{P}_{0}$ is rotation invariant.

In the proof of Theorem 4.1 we do not have to apply Lemma A.2 if the intensity function $f$ is strictly positive. In this case the following result holds.

Corollary 4.1. Let $X$ be a particle process with intensity measure as in (3.1). Furthermore, we assume that $X$ satisfies condition (A) and that its intensity function is strictly positive. Then the typical particle of $X$ is isotropic if and only if there exists a $z \in \mathbb{R}^{d}$ such that (4.1) holds for all $\mathcal{A} \in \mathcal{B}\left(\mathcal{K}_{0}\right)$ and $\vartheta \in S O_{d}$.

Proof. The above result follows directly from the proof of Theorem 4.1.

The stationarity of $X$ can be characterized in a similar way.

Theorem 4.2. Let $d \geq 2$ and let $X$ be a particle process with intensity measure as in (3.1). Furthermore, we assume that $X$ satisfies condition $(B)$. Then $X$ is weakly stationary if and only if

$$
\mathrm{E}_{\mathrm{P}_{0}} \mathbf{1}_{\mathcal{A}}(K) \int_{\mathbb{R}^{d}} f(z-\vartheta x) \Xi_{d-1}(K, \mathrm{~d} x \times A)=\mu_{z}(A, \mathcal{A})
$$

for all $z \in \mathbb{R}^{d}, A \in \mathcal{B}\left(S^{d-1}\right), \mathcal{A} \in \mathcal{B}\left(\mathcal{K}_{0}\right)$, and $\vartheta \in S O_{d}$. 
Example. Before we proceed with the proof of Theorem 4.2 let us first consider the case in which $d=1$. Here id is the only element of $S O_{1}$. Thus, any particle process on $\mathbb{R}^{1}$ satisfies (4.3) and Theorem 4.2 does not hold. An obvious analogous characterization is that $X$ is weakly stationary if and only if

$$
\mathrm{E}_{\mathrm{P}_{0}} \mathbf{1}_{\mathcal{A}}(K) \int_{\mathbb{R}^{d}} f(z+x) \Xi_{d-1}(K, \mathrm{~d} x \times A)=\mu_{z}(A, \mathcal{A})
$$

for all $z \in \mathbb{R}, A \in \mathcal{B}\left(S^{0}\right)$, and $\mathcal{A} \in \mathcal{B}\left(\mathcal{K}_{0}\right)$. Unfortunately, we can find the following counterexample. Let $X$ be a particle process on $\mathbb{R}$ with the property that its typical particle is almost surely a line segment (compact interval) $[-a, a]$ for some $a \in(0, \infty)$ and its intensity function $f$ is a nontrivial, nonnegative function of periodicity $2 a$. In this case the last equation holds for all $z \in \mathbb{R}, A \in \mathscr{B}\left(S^{0}\right)$, and $\mathcal{A} \in \mathscr{B}\left(\mathcal{K}_{0}\right)$ if and only if

$$
\mathbf{1}_{A}(-1) f(z+a)+\mathbf{1}_{A}(1) f(z-a)=\mathbf{1}_{A}(1) f(z+a)+\mathbf{1}_{A}(-1) f(z-a)
$$

for all $A \in \mathscr{B}\left(S^{0}\right)$. This is true by the periodicity of $f$, but $X$ is not necessarily weakly stationary. Also note that

$$
\mu_{z}(-A,-\mathcal{A})=\mu_{z}(A, \mathcal{A})
$$

for all $z \in \mathbb{R}^{1}, A \in \mathscr{B}\left(S^{0}\right)$, and $\mathcal{A} \in \mathcal{B}\left(\mathcal{K}_{0}\right)$; see Theorem 4.4, below.

Proof of Theorem 4.2. If $X$ is weakly stationary, i.e. $f \equiv \gamma>0$, (4.3) obviously holds for all $z \in \mathbb{R}^{d}$. Therefore, we assume that (4.3) holds. Written out, (4.3) means that

$$
\begin{aligned}
\mathrm{E}_{\mathrm{P}_{0}} \mathbf{1}_{\mathcal{A}}(K) \int_{\mathbb{R}^{d} \times S^{d-1}} f(z-\vartheta x) \mathbf{1}_{A}(u) \Xi_{d-1}(K, \mathrm{~d} x \times \mathrm{d} u) \\
\quad=\mathrm{E}_{\mathrm{P}_{0}} \mathbf{1}_{\mathcal{A}}(K) \int_{\mathbb{R}^{d} \times S^{d-1}} f(z-x) \mathbf{1}_{A}(u) \Xi_{d-1}(K, \mathrm{~d} x \times \mathrm{d} u)
\end{aligned}
$$

for all $z \in \mathbb{R}^{d}, \mathcal{A} \in \mathcal{B}\left(\mathcal{K}_{0}\right), A \in \mathcal{B}\left(S^{d-1}\right)$, and $\vartheta \in S O_{d}$. By the usual arguments from measure and integration theory this implies that, for any measurable function $g: S^{d-1} \rightarrow$ $[0, \infty)$,

$$
\begin{aligned}
\mathrm{E}_{\mathrm{P}_{0}} \mathbf{1}_{\mathcal{A}}(K) \int_{\mathbb{R}^{d} \times S^{d-1}} f(z-\vartheta x) g(u) \Xi_{d-1}(K, \mathrm{~d} x \times \mathrm{d} u) \\
\quad=\mathrm{E}_{\mathrm{P}_{0}} \mathbf{1}_{\mathcal{A}}(K) \int_{\mathbb{R}^{d} \times S^{d-1}} f(z-x) g(u) \Xi_{d-1}(K, \mathrm{~d} x \times \mathrm{d} u) .
\end{aligned}
$$

Let $g: S^{d-1} \rightarrow[0, \infty)$ be a continuous (and hence bounded) function. Since the above identity holds for all $\mathscr{A} \in \mathcal{B}\left(\mathcal{K}_{0}\right)$ and since, by weak continuity, the mapping

$$
K \mapsto \int_{\mathbb{R}^{d} \times S^{d-1}} f(z-\vartheta x) g(u) \Xi_{d-1}(K, \mathrm{~d} x \times \mathrm{d} u)
$$

is continuous for all $\vartheta \in S O_{d}$, we have, for all $K \in \operatorname{supp} \mathrm{P}_{0}, z \in \mathbb{R}^{d}$, and all $\vartheta \in S O_{d}$,

$$
\int_{\mathbb{R}^{d}} f(z-\vartheta x) g(u) \Xi_{d-1}(K, \mathrm{~d} x \times \mathrm{d} u)=\int_{\mathbb{R}^{d}} f(z-x) g(u) \Xi_{d-1}(K, \mathrm{~d} x \times \mathrm{d} u) .
$$

Since there exists a $K \in \operatorname{supp} \mathrm{P}_{0}$ that is strictly convex, the assertion follows from Lemma A.1 and Theorem A.1. 
For Poisson processes this immediately yields the following corollary.

Corollary 4.2. Let $d \geq 2$ and $X$ be a Poisson process with intensity measure as in (3.1). Furthermore, we assume that $X$ satisfies condition $(B)$. Then $X$ is stationary if and only if (4.3) holds for all $z \in \mathbb{R}^{d}, A \in \mathscr{B}\left(S^{d-1}\right), \mathcal{A} \in \mathcal{B}\left(\mathcal{K}_{0}\right)$, and $\vartheta \in S O_{d}$.

For the case in which $d=2$, the following more general results hold.

Theorem 4.3. Let $d=2$ and $X$ be a particle process with intensity measure as in (3.1). Furthermore, we assume that the typical particle of $X$ is almost surely two-dimensional. Then $X$ is weakly stationary if and only if (4.3) holds for all $z \in \mathbb{R}^{2}, A \in \mathscr{B}\left(S^{1}\right), \mathcal{A} \in \mathcal{B}\left(\mathcal{K}_{0}\right)$, and $\vartheta \in S O_{1}$.

Corollary 4.3. Let $d=2$ and $X$ be a Poisson process with intensity measure as in (3.1). Furthermore, we assume that the typical particle of $X$ is almost surely two-dimensional. Then $X$ is stationary if and only if (4.3) holds for all $z \in \mathbb{R}^{2}, A \in \mathscr{B}\left(S^{1}\right), \mathcal{A} \in \mathcal{B}\left(\mathcal{K}_{0}\right)$, and $\vartheta \in S_{2}$.

Proof of Theorem 4.3. From the proof of Theorem 4.2 it is obvious that we need only consider a process $X$ where each convex body $K \in \operatorname{supp} \mathrm{P}_{0}$ has the following property. Each $x \in \operatorname{reg} K$ lies in the relative interior of a line segment $S \subseteq$ bd $K$. Thus, let $x_{0} \in \operatorname{reg} K$ be a regular boundary point and let $S \subseteq$ bd $K$ be the line segment of maximal length with $x_{0} \in S$. Furthermore, let $u_{0} \in S^{d-1}$ be the outer normal vector of $K$ at $x_{0}$. Arguing as in the proof of Theorem 4.2 we obtain

$$
\int_{S} f(z-x) \mathscr{H}^{1}(\mathrm{~d} x)=\int_{S} f(z-\vartheta x) \mathscr{H}^{1}(\mathrm{~d} x)
$$

for all $\vartheta \in S O_{2}$ and $z \in \mathbb{R}^{2}$. Choosing suitable rotations the last identity can be used to show that there exists some $r>0$ such that, for all $z \in \mathbb{R}^{2}$ and all $z^{\prime} \in\left(z+2 r S^{1}\right)$, there exists a constant $c(z) \geq 0$ depending only on $z$ such that

$$
\int_{z^{\prime}+\vartheta(-S)} f(x) \mathscr{H}^{1}(\mathrm{~d} x)=c(z)
$$

for all $\vartheta \in \mathrm{SO}_{2}$. From this we can deduce that, for each rigid motion $g \in G_{d}$, the following holds:

$$
\int_{g(-S)} f(x) \mathscr{H}^{1}(\mathrm{~d} x)=c
$$

for a constant $c>0$. It immediately follows that there exists a constant $l>0$ such that $f(z)=f(z+l u)$ for all $z \in \mathbb{R}^{2}$ and $u \in S^{1}$. As in the proof of Theorem A.1, this implies that $f$ is constant.

Finally, we can state our main theorem.

Theorem 4.4. Let $d \geq 2$ and $X$ be a particle process with intensity measure as in (3.1). Furthermore, we assume that $X$ satisfies conditions $(A)$ and $(B)$. Then $X$ is weakly stationary and weakly isotropic if and only if

$$
\mu_{z}(\vartheta A, \vartheta \mathcal{A})=\mu_{z}(A, \mathcal{A})
$$

for all $z \in \mathbb{R}^{d}, A \in \mathscr{B}\left(S^{d-1}\right), \mathcal{A} \in \mathcal{B}\left(\mathcal{K}_{0}\right)$, and $\vartheta \in S O_{d}$. 
Let $X$ be a particle process that satisfies the assumptions of Theorem 4.4. Since both $f$ and $\mathrm{P}_{0}$ are uniquely determined, weak stationarity and weak isotropy of $X$ imply that $f$ is a constant function and $\mathrm{P}_{0}$ is rotation invariant. Hence, from Theorems 4.1, 4.2, and 4.4 we obtain

$$
X \text { satisfies (4.1) and (4.3) } \Longleftrightarrow X \text { satisfies (4.4). }
$$

Unfortunately, a direct proof of this equivalence does not seem to be possible.

Proof of Theorem 4.4. If $X$ is weakly stationary and weakly isotropic, (4.4) obviously holds. Therefore, let us assume that (4.4) holds. Written out, (4.4) means that

$$
\begin{aligned}
\mathrm{E}_{\mathrm{P}_{0}} \mathbf{1}_{\vartheta \mathcal{A}}(K) \int_{\mathbb{R}^{d}} f(z-x) \Xi_{d-1}(K, \mathrm{~d} x \times \vartheta A) \\
\quad=\mathrm{E}_{\mathrm{P}_{0}} \mathbf{1}_{\mathcal{A}}(K) \int_{\mathbb{R}^{d}} f(z-x) \Xi_{d-1}(K, \mathrm{~d} x \times A) .
\end{aligned}
$$

First, choose $A=S^{d-1}$. As in the proof of Theorem 4.1, we can show that, for all $\vartheta \in S O_{d}$, there exists a function $\eta_{\vartheta}: \mathcal{K}_{0} \rightarrow \mathcal{K}_{0}$ such that $\mathrm{E}_{\mathrm{P}_{0}} g(K)=\mathrm{E}_{\mathrm{P}_{0}} g(\vartheta K) \eta_{\vartheta}(\vartheta K)$ for all measurable mappings $g: \mathcal{K}_{0} \rightarrow[0, \infty)$. From (4.4) it follows that

$$
\begin{aligned}
\mathrm{E}_{\mathrm{P}_{0}} \mathbf{1}_{\vartheta \mathcal{A}}(K) \int_{\mathbb{R}^{d}} f(z-x) \Xi_{d-1}(K, \mathrm{~d} x \times \vartheta A) \\
=\mathrm{E}_{\mathrm{P}_{0}} \mathbf{1}_{\mathcal{A}}(K) \int_{\mathbb{R}^{d}} f(z-x) \Xi_{d-1}(K, \mathrm{~d} x \times A) \quad(\text { by }(4.4)) \\
=\mathrm{E}_{\mathrm{P}_{0}} \mathbf{1}_{\mathcal{A}}\left(\vartheta^{-1} K\right) \int_{\mathbb{R}^{d}} f(z-x) \Xi_{d-1}\left(\vartheta^{-1} K, \mathrm{~d} x \times A\right) \eta_{\vartheta^{-1}}\left(\vartheta^{-1} K\right) \\
=\mathrm{E}_{\mathrm{P}_{0}} \mathbf{1}_{\vartheta \mathcal{A}}(K) \int_{\mathbb{R}^{d}} f\left(z-\vartheta^{-1} x\right) \Xi_{d-1}(K, \mathrm{~d} x \times \vartheta A) \eta_{\vartheta-1}\left(\vartheta^{-1} K\right) .
\end{aligned}
$$

Hence, for all $z \in \mathbb{R}^{d}, A \in \mathcal{B}\left(S^{d-1}\right), \vartheta \in S O_{d}$, and $\mathrm{P}_{0}$-almost all $K \in \mathcal{K}_{0}$, we have

$$
\int_{\mathbb{R}^{d}} f\left(z-\vartheta^{-1} x\right) \Xi_{d-1}(K, \mathrm{~d} x \times A) \eta_{\vartheta^{-1}}\left(\vartheta^{-1} K\right)=\int_{\mathbb{R}^{d}} f(z-x) \Xi_{d-1}(K, \mathrm{~d} x \times A) .
$$

As before let $\mathcal{K}_{0}^{(n)}:=\left\{K \in \mathcal{K}_{0} \mid \operatorname{dim} K \in\{d-1, d\}, K \subseteq n B^{d}\right\}$ and $r(n)$ be as defined in Lemma A.2. Since (4.5) holds for all $z \in \mathbb{R}^{d}$, choosing $A=S^{d-1}$ implies that, for all $n \in \mathbb{N}$ and $\mathrm{P}_{0}$-almost all $K \in \mathcal{K}_{0}^{(n)}$,

$$
\eta_{\vartheta^{-1}}\left(\vartheta^{-1} K\right)=\underbrace{\frac{\overbrace{\int_{\mathbb{R}^{d}} \int_{r(n) B^{d}} f(z-x) \lambda_{d}(\mathrm{~d} z) C_{d-1}(K, \mathrm{~d} x)}^{>0}}{\underbrace{}_{\mathbb{R}^{d} \int_{r(n) B^{d}} f\left(z-\vartheta^{-1} x\right) \lambda_{d}(\mathrm{~d} z) C_{d-1}(K, \mathrm{~d} x)}} .}_{>0}
$$

For fixed $z \in \mathbb{R}^{d}$ and $\vartheta \in S O_{d}$, the mappings

$$
x \mapsto f(z-x) \text { and } \quad x \mapsto f\left(z-\vartheta^{-1} x\right)
$$


are continuous. Furthermore, for all $K \in \mathcal{K}_{0}^{(n)}, z \in r(n) B^{d}$, and $x \in$ bd $K$, we have

$$
f(z-x) \leq \max _{y \in(r(n)+n) B^{d}} f(y)<\infty \quad \text { and } \quad f\left(z-\vartheta^{-1} x\right) \leq \max _{y \in(r(n)+n) B^{d}} f(y)<\infty .
$$

Hence, by dominated convergence the mappings

$$
x \mapsto \int_{r(n) B^{d}} f(z-x) \lambda_{d}(\mathrm{~d} z) \quad \text { and } \quad x \mapsto \int_{r(n) B^{d}} f\left(z-\vartheta^{-1} x\right) \lambda_{d}(\mathrm{~d} z)
$$

are continuous for each $n \in \mathbb{N}$. This immediately yields the continuity of the mappings

$$
K \mapsto \int_{\mathbb{R}^{d}} \int_{r(n) B^{d}} f(z-x) \lambda_{d}(\mathrm{~d} z) C_{d-1}(K, \mathrm{~d} x)
$$

and

$$
K \mapsto \int_{\mathbb{R}^{d}} \int_{r(n) B^{d}} f\left(z-\vartheta^{-1} x\right) \lambda_{d}(\mathrm{~d} z) C_{d-1}(K, \mathrm{~d} x)
$$

on $\mathcal{K}_{0}^{(n)}$. Since this is true for each $n \in \mathbb{N}$ by (4.6), the function $\eta_{\vartheta}$ is equal to a continuous function for $\mathrm{P}_{0}$-almost all $K \in \mathcal{K}_{0}$. So, without loss of generality, we can assume that $\eta_{\vartheta}$ is a continuous function on $\mathcal{K}_{0}$. As in the proof of Theorem 4.2 we can then show that, for all $z \in \mathbb{R}^{d}, \vartheta \in S O_{d}$, continuous (and bounded) $g: S^{d-1} \rightarrow[0, \infty)$, and $K \in \operatorname{supp} \mathrm{P}_{0}$, we have

$$
\begin{gathered}
\int_{\mathbb{R}^{d} \times S^{d-1}} f\left(z-\vartheta^{-1} x\right) g(u) \Xi_{d-1}(K, \mathrm{~d} x \times \mathrm{d} u) \eta_{\vartheta^{-1}}\left(\vartheta^{-1} K\right) \\
=\int_{\mathbb{R}^{d} \times S^{d-1}} f(z-x) g(u) \Xi_{d-1}(K, \mathrm{~d} x \times \mathrm{d} u) .
\end{gathered}
$$

Since there exists a $K \in \operatorname{supp} \mathrm{P}_{0}$ that is strictly convex, $f$ is constant by Lemma A.1 and Theorem A.1. From (4.6) we obtain $\eta_{\vartheta^{-1}}\left(\vartheta^{-1} K\right)=1$ for each $\vartheta \in S O_{d}$ and $\mathrm{P}_{0^{-}}$-almost all $K \in \mathcal{K}_{0}$. This implies that $\mathrm{P}_{0}$ is rotation invariant and, hence, that $X$ is weakly stationary and weakly isotropic.

We can rephrase Theorem 4.4 as follows.

Corollary 4.4. Let $d \geq 2$ and $X$ be a particle process with intensity measure as in (4.1). Furthermore, we assume that $X$ satisfies conditions $(A)$ and $(B)$. Then $X$ is weakly stationary and weakly isotropic if and only if $\mu_{z}$ is rotation invariant for all $z \in \mathbb{R}^{d}$.

Proof. The assertion follows directly from Theorem 4.4.

For Poisson processes this yields the following corollary.

Corollary 4.5. Let $d \geq 2$ and $X$ be a Poisson process with intensity measure as in (4.1). Furthermore, we assume that $X$ satisfies conditions $(A)$ and $(B)$. Then $X$ is stationary and isotropic if and only if $\mu_{z}$ is rotation invariant for all $z \in \mathbb{R}^{d}$.

\section{Processes of cylinders}

In this section we want to combine our Theorem 4.4 and Theorem 1 of [6] to obtain a similar result for processes of cylinders.

Let $q \in\{0, \ldots, d-1\}$. We define a cylinder $Z$ with direction space $L \in \mathcal{L}_{q}^{d}$ and basis $K \in \mathcal{K}^{\prime}, K \subseteq L^{\perp}$, as the set $Z:=K+L$, i.e. the Minkowski (vector) sum of $K$ and $L$. 
Let $\mathcal{Z}_{q}^{d}$ be the set of all cylinders with $q$-dimensional direction space and let $\mathcal{Z}_{q, 0}^{d} \subseteq \mathcal{Z}_{q}^{d}$ be the subset of all cylinders with a basis $K$ from $\mathcal{K}_{0}$. Both $\mathcal{Z}_{q}^{d}$ and $\mathcal{Z}_{q, 0}^{d}$ are Borel subsets of $\mathcal{F}^{\prime}$. Let $l \in\{1, \ldots, d\}$ and $m \in\{0, \ldots, l\}$. Throughout this section $X_{l, m}$ will denote a cylinder process on $\mathcal{Z}_{d-l}^{d}$ with nontrivial locally finite intensity measure $\Theta$. Furthermore, we assume that $\Theta$ is of the form

$$
\Theta(\mathcal{A})=\int_{\mathcal{L}_{d-l}^{d}} \int_{\mathcal{K}_{0}} \int_{L^{\perp}} \mathbf{1}_{\mathcal{A}}(K+L+x) f(L+x) \lambda_{L^{\perp}}(\mathrm{d} x) \mathrm{P}(L, \mathrm{~d} K) \Phi(\mathrm{d} L),
$$

$\mathcal{A} \in \mathscr{B}\left(\mathcal{Z}_{d-l}^{d}\right)$. Here, $f: \mathcal{E}_{d-l}^{d} \rightarrow[0, \infty)$ denotes a continuous function; for each $L \in \mathcal{L}_{d-l}^{d}$, $\mathrm{P}(L, \cdot)$ is a probability measure on $\mathcal{K}_{0}$ with the property that

$$
\mathrm{P}\left(L,\left\{K \in \mathcal{K}_{0} \mid \operatorname{dim} K=m, K \subseteq L^{\perp}\right\}\right)=1 ;
$$

for all $\mathcal{A} \in \mathscr{B}\left(\mathcal{K}_{0}\right)$, the mapping $L \mapsto \mathrm{P}(L, \mathcal{A})$ is measurable; and $\Phi$ is a finite measure on $\mathcal{L}_{d-l}^{d}$. Note that $f, \mathrm{P}(L, \cdot)$, and $\Phi$ are not uniquely determined by $\Theta$. We can consider $X_{l, m}$ as a process of cylinders for which $\Phi$ describes the distribution of the direction spaces and $\mathrm{P}(L, \cdot)$ the distribution of the bases. Note that $X_{l, m}$ is a process of convex particles if $l=d$ and $m>0$ and that $X_{l, m}$ is a process of $(d-l)$-flats if $m=0$. For the rest of the paper we assume that $(l, m) \neq(d, 0)$.

Before generalizing Theorem 1 of [6], we introduce our main object of study, the generalized local mean normal measures for a cylinder process. For all $z \in \mathbb{R}^{d}$, we therefore define a set function $\mu_{z}: \mathcal{B}\left(\mathcal{Z}_{d-l, 0}^{d}\right) \times \mathscr{B}\left(S^{d-1}\right)$ by

$$
\begin{aligned}
\mu_{z}(\mathcal{A}, A):= & \int_{\mathscr{L}_{d-l}^{d}} \int_{\mathcal{K}_{0}} \mathbf{1}_{\mathcal{A}}(K+L) \\
& \times \int_{\operatorname{rel~bd} K} \mathbf{1}_{A}\left(\tilde{\sigma}_{K}(x)\right) f(L+z-x) \mathscr{H}^{m-1}(\mathrm{~d} x) \mathrm{P}(L, \mathrm{~d} K) \Phi(\mathrm{d} L),
\end{aligned}
$$

$\mathcal{A} \in \mathcal{B}\left(\mathcal{Z}_{d-l, 0}^{d}\right)$ and $A \in \mathscr{B}\left(S^{d-1}\right)$. If $m=0$, we define

$$
\int_{\text {rel bd } K} \mathbf{1}_{A}\left(\tilde{\sigma}_{K}(x)\right) f(L+z-x) \mathscr{H}^{m-1}(\mathrm{~d} x):=1
$$

for all $A \in \mathscr{B}\left(S^{d-1}\right)$. Note that $\tilde{\sigma}_{K}(x)=\tilde{\sigma}_{K+L}(x) \cap$ aff $K$.

Remark. Let $B \in \mathscr{B}\left(\mathbb{R}^{d}\right)$ be bounded. For $A \in \mathscr{B}\left(S^{d-1}\right)$ and $K+L \in \mathcal{Z}_{d-l}^{d}$ with $\operatorname{dim} K=$ $m \leq l$ and $K \subseteq L^{\perp}$, we have

$$
\mathscr{H}^{d-l+m-1}\left(B \cap\left(\tilde{\sigma}_{K}^{-1}(A)+L\right)\right)=\int_{L} \int_{\operatorname{rel~bd} K} \mathbf{1}_{B}(a+b) \mathbf{1}_{A}\left(\tilde{\sigma}_{K}(a)\right) \mathscr{H}^{m-1}(\mathrm{~d} a) \lambda_{L}(\mathrm{~d} b) .
$$

By Lemma B.2, Theorem 1.1.7 of [8], and monotone convergence the mapping

$$
K+L \mapsto \int_{L} \int_{\operatorname{rel~bd} K} \mathbf{1}_{B}(a+b) \mathbf{1}_{A}\left(\tilde{\sigma}_{K}(a)\right) \mathscr{H}^{m-1}(\mathrm{~d} a) \lambda_{L}(\mathrm{~d} b)
$$

is measurable. The latter (together with Lemma B.2 and respective theorems from [5], [7], and [8]) implies the measurability of the mappings we use in this section. 
Let us state some properties of $\mu_{z}$.

Theorem 5.1. Let $l \in\{1, \ldots, d\}, m \in\{0, \ldots, l\}$, and $X_{l, m}$ be a particle process with intensity measure as in (5.1), and let $\mu_{z}$ be as defined above. Then the set function

$$
\mathscr{B}\left(\mathcal{Z}_{d-l, 0}^{d}\right) \times \mathscr{B}\left(S^{d-1}\right) \rightarrow[0, \infty),(\mathcal{A}, A) \mapsto \mu_{z}(\mathcal{A}, A),
$$

can be extended to a measure on $\mathcal{B}\left(\mathcal{Z}_{d-l, 0}^{d}\right) \times \mathcal{B}\left(S^{d-1}\right)$. For $\lambda_{d}$-almost all $z \in \mathbb{R}^{d}$, the measure is finite and uniquely determined by $\Theta$.

Proof. Let $\mathcal{A} \in \mathscr{B}\left(\mathcal{Z}_{d-l, 0}^{d}\right)$ and $A \in \mathscr{B}\left(S^{d-1}\right)$. The first assertion is obvious because we can rewrite $\mu_{z}$ in terms of $\mathbf{1}_{A \times \mathcal{A}}$. For $B \in \mathscr{B}\left(\mathbb{R}^{d}\right)$ bounded, we have, by Campbell's theorem,

$$
\begin{aligned}
\mathrm{E} \quad \sum_{(K+L) \in X_{l, m}} \mathbf{1}_{\mathcal{A}}(K+L-s(K)) \mathcal{H}^{d-l+m-1}\left(B \cap\left(\tilde{\sigma}_{K}^{-1}(A)+L\right)\right) \\
=\int_{\mathcal{L}_{d-l}^{d}} \int_{\mathcal{K}_{0}} \mathbf{1}_{\mathcal{A}}(K+L) \int_{L^{\perp}} \int_{L} \int_{\text {rel bd } K} \mathbf{1}_{B-z}(a+b) \mathbf{1}_{A}\left(\tilde{\sigma}_{K}(a)\right) \mathscr{H}^{m-1}(\mathrm{~d} a) \lambda_{L}(\mathrm{~d} b) \\
\times f(L+z) \lambda_{L^{\perp}}(\mathrm{d} z) \mathrm{P}(L, \mathrm{~d} K) \Phi(\mathrm{d} L) \\
=\int_{\mathcal{L}_{d-l}^{d}} \int_{\mathcal{K}_{0}} \mathbf{1}_{\mathcal{A}}(K+L) \int_{L^{\perp}} \int_{L} \int_{\operatorname{rel~bd} K} \mathbf{1}_{B}(b+z) \mathbf{1}_{A}\left(\tilde{\sigma}_{K}(a)\right) f(L+z-a) \mathscr{H}^{m-1}(\mathrm{~d} a) \\
=\int_{\mathbb{R}^{d}} \mathbf{1}_{B}(z) \mu_{z}(\mathcal{A}, A) \lambda_{d}(\mathrm{~d} z) .
\end{aligned}
$$

Hence, for $\lambda_{d}$-almost all $z \in \mathbb{R}^{d}$, the measure $\mu_{z}$ is uniquely determined by $\Theta$. Furthermore, we have

$$
\begin{aligned}
& \mathrm{E} \sum_{(K+L) \in X_{l, m}} \mathbf{1}_{\mathcal{Z}_{d-l, 0}^{d}}(K+L-s(K)) \mathscr{H}^{d-l+m-1}\left(B \cap\left(\tilde{\sigma}_{K}^{-1}\left(S^{d-1}\right)+L\right)\right) \\
& =\mathrm{E} \sum_{(K+L) \in X_{l, m}} \mathscr{H}^{d-l+m-1}(B \cap \operatorname{rel~bd}(K+L)) \\
& =c \mathrm{E} \sum_{(K+L) \in X_{l, m}} C_{d-l+m-1}(K+L, B)
\end{aligned}
$$

for some constant $c>0$ depending only on $d$ and $d-l+m$. Here, $C_{d-l+m-1}(K+L, \cdot)$ denotes the curvature measure of $K+L$ (extended to arbitrary closed convex sets). By Theorem 4.2 of [1] the Borel measure

$$
\mathrm{E} \sum_{(K+L) \in X_{l, m}} C_{d-l+m-1}(K+L, \cdot)
$$

on $\mathbb{R}^{d}$ is locally finite and, thus, $\mu_{z}$ is finite for $\lambda_{d}$-almost all $z \in \mathbb{R}^{d}$.

Note that, for $l=m=d$, the above definition of $\mu_{z}(\mathcal{A}, A)$ matches the definition given in Section 3. Furthermore, $X_{l, m}$ is a translation-regular $(d-l)$-flat process with continuous density as introduced in [6] if $m=0$. In this case $\mu_{z}$ coincides with the direction measure $\varphi(z, \cdot)$ defined in [6, p. 142]. As before, we call $\mu_{z}$ the generalized local mean normal measure of $X_{l, m}$ at $z$. We now present the following generalization of our Theorem 4.4 and Theorem 1 of [6]. 
Theorem 5.2. Let $d \geq 2, l \in\{1, \ldots, d\}$, and $m \in\{0, \ldots, l\}$, and let $X_{l, m}$ be a cylinder process with intensity measure as in (5.1). Furthermore, we assume that there exist $L \in \operatorname{supp} \Phi$ and $K \in \operatorname{supp} \mathrm{P}(L, \cdot)$ such that $K$ is strictly convex in aff $K$. Then $X_{l, m}$ is weakly stationary and weakly isotropic if and only if

$$
\mu_{z}(\vartheta \mathcal{A}, \vartheta A)=\mu_{z}(\mathcal{A}, A)
$$

for all $z \in \mathbb{R}^{d}, \mathcal{A} \in \mathcal{B}\left(\mathcal{Z}_{d-l, 0}^{d}\right), A \in \mathcal{B}\left(S^{d-1}\right)$, and $\vartheta \in S O_{d}$.

Proof. If $X_{l, m}$ is weakly stationary and weakly isotropic it is easy to see that (5.2) holds. Therefore, we assume that (5.2) holds. If $m=0$ the assertion follows from Theorem 1 of [6]. So, let $m \in\{1, \ldots, l\}$. The case in which $l=d$ can be proved in the same way as Theorem 4.4, i.e. using Theorem A.1. Thus, let $1 \leq l \leq d-1$. First, we consider the case in which $m \geq 2$. Lemma B. 2 states that the mapping

$$
K+L \mapsto \int_{\text {rel bd } K} g\left(\tilde{\sigma}_{K}(x)\right) f(L+z-x) \mathscr{H}^{m-1}(\mathrm{~d} x)
$$

is continuous for all continuous functions $g: S^{d-1} \rightarrow[0, \infty)$ and $z \in \mathbb{R}^{d}$. As in the respective proofs of Theorems 4.1 and 4.4 , by choosing $A=S^{d-1}$ we can show that, for all $\vartheta \in S O_{d}$, the measure $\mathrm{P}(L, \cdot) \otimes \Phi$ is absolutely continuous with respect to $\vartheta \circ(\mathrm{P}(L, \cdot) \otimes \Phi)$ and we can find a continuous density function. Furthermore, it is easy to see that if $K \in \operatorname{supp} \mathrm{P}(L, \cdot)$ for some $L \in \operatorname{supp} \Phi$ then $\vartheta L \in \operatorname{supp} \Phi$ and $\vartheta K \in \operatorname{supp} \mathrm{P}(\vartheta L, \cdot)$ for all $\vartheta \in S O_{d}$. This implies that $\operatorname{supp} \Phi=\mathcal{L}_{d-l}^{d}$.

By (5.2) we have, for all $z \in \mathbb{R}^{d}, \mathcal{A} \in \mathscr{B}\left(\mathcal{Z}_{d-l, 0}^{d}\right), A \in \mathscr{B}\left(S^{d-1}\right)$, and $\vartheta \in S O_{d}$,

$$
\begin{aligned}
& \int_{\mathscr{L}_{d-l}^{d}} \int_{\mathcal{K}_{0}} \mathbf{1}_{\vartheta \mathcal{A}}(K+L) \int_{\text {rel bd } K} \mathbf{1}_{\vartheta A}\left(\tilde{\sigma}_{K}(x)\right) f(L+z-x) \mathcal{H}^{m-1}(\mathrm{~d} x) \mathrm{P}(L, \mathrm{~d} K) \Phi(\mathrm{d} L) \\
& =\int_{\mathscr{L}_{d-l}^{d}} \int_{\mathcal{K}_{0}} \mathbf{1}_{\mathcal{A}}(K+L) \\
& \times \int_{\text {rel bd } K} \mathbf{1}_{A}\left(\tilde{\sigma}_{K}(x)\right) f(L+z-x) \mathscr{H}^{m-1}(\mathrm{~d} x) \mathrm{P}(L, \mathrm{~d} K) \Phi(\mathrm{d} L) \quad(\text { by }(5.2)) \\
& =\int_{\mathcal{L}_{d-l}^{d}} \int_{\mathcal{K}_{0}} \mathbf{1}_{\mathcal{A}}\left(\vartheta^{-1}(K+L)\right) \\
& \times \int_{\operatorname{rel~bd}\left(\vartheta^{-1} K\right)} \mathbf{1}_{A}\left(\tilde{\sigma}_{\vartheta^{-1} K}(x)\right) f\left(\vartheta^{-1} L+z-x\right) \\
& \times \mathscr{H}^{m-1}(\mathrm{~d} x) \eta_{\vartheta^{-1}}\left(\vartheta^{-1}(K+L)\right) \mathrm{P}(L, \mathrm{~d} K) \Phi(\mathrm{d} L) \\
& =\int_{\mathscr{L}_{d-l}^{d}} \int_{\mathcal{K}_{0}} \mathbf{1}_{\vartheta \mathcal{A}}(K+L) \int_{\text {rel bd } K} \mathbf{1}_{\vartheta A}\left(\tilde{\sigma}_{K}(x)\right) f\left(\vartheta^{-1} L+z-\vartheta^{-1} x\right) \mathscr{H}^{m-1}(\mathrm{~d} x) \\
& \times \eta_{\vartheta^{-1}}\left(\vartheta^{-1}(K+L)\right) \mathrm{P}(L, \mathrm{~d} K) \Phi(\mathrm{d} L) .
\end{aligned}
$$

For the last equation we used the rotation invariance of the Hausdorff measure. The above is 
equivalent to

$$
\begin{gathered}
\int_{\mathscr{L}_{d-l}^{d}} \int_{\mathcal{K}_{0}} \mathbf{1}_{\mathcal{A}}(K+L) \int_{\text {rel bd } K} \mathbf{1}_{A}\left(\tilde{\sigma}_{K}(x)\right) f(L+z-x) \mathcal{H}^{m-1}(\mathrm{~d} x) \mathrm{P}(L, \mathrm{~d} K) \Phi(\mathrm{d} L) \\
=\int_{\mathcal{L}_{d-l}^{d}} \int_{\mathcal{K}_{0}} \mathbf{1}_{\mathcal{A}}(K+L) \int_{\text {rel bd } K} \mathbf{1}_{A}\left(\tilde{\sigma}_{K}(x)\right) f(\vartheta L+z-\vartheta x) \mathcal{H}^{m-1}(\mathrm{~d} x) \\
\times \eta_{\vartheta}(\vartheta(K+L)) \mathrm{P}(L, \mathrm{~d} K) \Phi(\mathrm{d} L)
\end{gathered}
$$

for all $z \in \mathbb{R}^{d}, \mathcal{A} \in \mathscr{B}\left(\mathcal{Z}_{d-l, 0}^{d}\right), A \in \mathscr{B}\left(S^{d-1}\right)$, and $\vartheta \in S O_{d}$. As in the proof of Theorem 4.2, we can show that, for all $K \in \operatorname{supp} \mathrm{P}(L, \cdot)$ with $L \in \operatorname{supp} \Phi, z \in \mathbb{R}^{d}, \vartheta \in S O_{d}$, and continuous functions $g: S^{d-1} \rightarrow[0, \infty)$, we have

$$
\begin{aligned}
\int_{\text {rel bd } K} g\left(\tilde{\sigma}_{K}(x)\right) f(L+z-x) \mathscr{H}^{m-1}(\mathrm{~d} x) \\
=\int_{\text {rel bd } K} g\left(\tilde{\sigma}_{K}(x)\right) f(\vartheta L+z-x) \mathcal{H}^{m-1}(\mathrm{~d} x) \eta_{\vartheta}(\vartheta(K+L)) .
\end{aligned}
$$

Since one of the $K \mathrm{~s}$ was assumed to be strictly convex in aff $K$ and $\operatorname{dim} K=\operatorname{dim}$ aff $K>1$ it follows, from Theorem A.1 (using rotations $\vartheta \in S O_{d}$ with $\vartheta L=L$ ), that, for all $z \in \mathbb{R}^{d}$ and $L \in \mathcal{L}_{d-l}^{d}$, we have $f(L+z)=f(L)$. Choosing $A=S^{d-1}$ we obtain, from (5.3), for all $\mathcal{A} \in \mathcal{B}\left(\mathcal{Z}_{d-l, 0}^{d}\right)$,

$$
\begin{aligned}
\int_{\mathscr{L}_{d-l}^{d}} & \int_{\mathcal{K}_{0}} \mathbf{1}_{\mathcal{A}}(K+L) f(L) \mathscr{H}^{m-1}(K) \mathrm{P}(L, \mathrm{~d} K) \Phi(\mathrm{d} L) \\
= & \int_{\mathscr{L}_{d-l}^{d}} \int_{\mathcal{K}_{0}} \mathbf{1}_{\vartheta^{-1} \mathcal{A}}(K+L) f(\vartheta L) \eta_{\vartheta}(\vartheta(K+L)) \mathscr{H}^{m-1}(K) \mathrm{P}(L, \mathrm{~d} K) \Phi(\mathrm{d} L) \\
= & \int_{\mathscr{L}_{d-l}^{d}} \int_{\mathcal{K}_{0}} \mathbf{1}_{\vartheta^{-1} \mathcal{A}}(K+L) f(L) \mathscr{H}^{m-1}(K) \mathrm{P}(L, \mathrm{~d} K) \Phi(\mathrm{d} L) .
\end{aligned}
$$

Note that $\mathscr{H}^{m-1}$ is rotation invariant. Hence, for $(\mathrm{P}(L, \cdot) \otimes \Phi)$-almost all $K+L \in \mathcal{Z}_{d-l, 0}^{d}$, we have $f(\vartheta L) \eta_{\vartheta}(\vartheta(K+L))=f(L)$. Let $\mathcal{A} \in \mathcal{B}\left(\mathcal{Z}_{d-l}^{d}\right)$ and $\vartheta \in S O_{d}$. As

$$
\begin{aligned}
\Theta(\vartheta \mathcal{A})= & \int_{\mathcal{L}_{d-l}^{d}} \int_{\mathcal{K}_{0}} \int_{L^{\perp}} \mathbf{1}_{\mathcal{A}}\left(\vartheta^{-1}(K+L+x)\right) f(L) \lambda_{L^{\perp}}(\mathrm{d} x) \mathrm{P}(L, \mathrm{~d} K) \Phi(\mathrm{d} L) \\
= & \int_{\mathcal{L}_{d-l}^{d}} \int_{\mathcal{K}_{0}} \int_{L^{\perp}} \mathbf{1}_{\mathcal{A}}\left(K+L+\vartheta^{-1} x\right) f(\vartheta L) \eta_{\vartheta}(\vartheta(K+L)) \\
& \times \lambda_{(\vartheta L)^{\perp}}(\mathrm{d} x) \mathrm{P}(L, \mathrm{~d} K) \Phi(\mathrm{d} L) \\
= & \Theta(\mathcal{A}),
\end{aligned}
$$

the intensity measure $\Theta$ is rotation invariant. Invariance under translations follows analogously. Now let $m=1$ and, without loss of generality, $l=1$ (for $l \in\{2, \ldots, d\}$, the following argument can easily be adapted). For all $\vartheta \in S O_{d}$, there exists a continuous mapping

$$
\eta_{\vartheta}: \mathcal{Z}_{d-1,0}^{d} \rightarrow[0, \infty)
$$


such that

$$
\begin{gathered}
\int_{\mathcal{L}_{d-1}^{d}} \int_{\mathcal{K}_{0}} \mathbf{1}_{\mathcal{A}}(K+L) \int_{\text {rel bd } K} \mathbf{1}_{A}\left(\tilde{\sigma}_{K}(x)\right) f(L+z-x) \mathcal{H}^{0}(\mathrm{~d} x) \mathrm{P}(L, \mathrm{~d} K) \Phi(\mathrm{d} L) \\
=\int_{\mathcal{L}_{d-1}^{d}} \int_{\mathcal{K}_{0}} \mathbf{1}_{\mathcal{A}}(K+L) \int_{\text {rel bd } K} \mathbf{1}_{A}\left(\tilde{\sigma}_{K}(x)\right) f(\vartheta L+z-x) \mathscr{H}^{0}(\mathrm{~d} x) \eta_{\vartheta}(\vartheta(K+L)) \\
\times \mathrm{P}(L, \mathrm{~d} K) \Phi(\mathrm{d} L) .
\end{gathered}
$$

Again, for all $K \in \operatorname{supp} \mathrm{P}(L, \cdot)$ with $L \in \operatorname{supp} \Phi, z \in \mathbb{R}^{d}, \vartheta \in S O_{d}$, and continuous functions $g: S^{d-1} \rightarrow[0, \infty)$, we have

$$
\begin{aligned}
\int_{\text {rel bd } K} g\left(\tilde{\sigma}_{K}(x)\right) f(L+z-x) \mathscr{H}^{0}(\mathrm{~d} x) \\
=\int_{\text {rel bd } K} g\left(\tilde{\sigma}_{K}(x)\right) f(\vartheta L+z-x) \mathscr{H}^{0}(\mathrm{~d} x) \eta_{\vartheta}(\vartheta(K+L)) .
\end{aligned}
$$

Let $K \in \operatorname{supp} \mathrm{P}(L, \cdot)$ for $L \in \operatorname{supp} \Phi$. As $K \in \mathcal{K}_{0}$ and $\operatorname{dim} K=1, K$ is a line segment in $L^{\perp}$ with midpoint 0 . We define $l=l(K):=\frac{1}{2} \mathscr{H}^{1}(K)>0$. Also, let $u=u(K) \in S^{d-1}$ be a unit vector such that $L^{\perp}=\{\alpha u \mid \alpha \in \mathbb{R}\}$. In particular, we have $K=[-l u, l u]$. Here, for any $x, y \in \mathbb{R}^{d},[x, y]$ denotes the line segment between $x$ and $y$. Furthermore, let $\vartheta \in S O_{d}$ be a rotation with the property that $\vartheta L=L$ and $\vartheta u=-u$. From (5.4) we obtain, by choosing $g \equiv 1$,

$$
f(L+z+l u)+f(L+z-l u)=\eta_{\vartheta}(\vartheta(K+L))(f(L+z+l u)+f(L+z-l u)),
$$

i.e. $\eta_{\vartheta}(\vartheta(K+L))=1$. Note that we chose $z$ such that $f(L+z+l u)+f(L+z-l u)>0$. Let $z \in \mathbb{R}^{d}$ be arbitrary and let $g: S^{d-1} \rightarrow[0, \infty)$ be a continuous function with the property that $g(u)=1$ and $g(-u)=0$. Using the same rotation $\vartheta$ as before (which has the property that $\left.\eta_{\vartheta}(\vartheta(K+L))=1\right)$, we obtain

$$
f(L+z+l u)=f(L+z-l u) .
$$

Hence, $f(L+\cdot)$ has periodicity $2 l$. Combining (5.4) and (5.5) with a suitable choice of $g$, i.e. a $g$ that is continuous with the property that $g(u)=g(\vartheta u)=1$ and $g(-u)=g(-\vartheta u)=0$, yields, for all $\vartheta \in S O_{d}$ and $z \in \mathbb{R}^{d}$,

$$
\begin{aligned}
\eta_{\vartheta}(\vartheta(K+L)) f(\vartheta(L+l u)+z+2 l u) & =f(L+l u+z+2 l u) \\
& =f(L+z+l u) \\
& =\eta_{\vartheta}(\vartheta(K+L)) f(\vartheta(L+l u)+z),
\end{aligned}
$$

i.e.

$$
f(\vartheta(L+l u)+z+2 l u)=f(\vartheta(L+l u)+z) .
$$

Obviously, for all $\alpha \in[0,2 l]$, we can find $\vartheta \in S O_{d}$ such that $\vartheta L+2 l u=\vartheta L+\alpha \vartheta u$. In combination with (5.3) this yields

$$
f(\vartheta L+z+l \vartheta u+\alpha \vartheta u)=f(\vartheta L+z+l \vartheta u) .
$$

The last equation is equivalent to $f(L+z+\alpha u)=f(L+z)$ for all $z \in L^{\perp}$ and $\alpha \in[0,2 l]$. Therefore, the function $f$ can only depend on $L$. The translation and rotation invariance of $\Theta$ follow as before. 
Remark. Theorem 1 of [6] and Theorem 4.4 are included in Theorem 5.2 as special cases. Theorem 1 of [6] follows by choosing $m=0$ and Theorem 4.4 follows for $l=m=d$.

Finally, let us state two corollaries of Theorem 5.2. The first is a reformulation of Theorem 5.2 and the second is a specialization to Poisson processes.

Corollary 5.1. Let $d \geq 2, l \in\{1, \ldots, d\}$, and $m \in\{0, \ldots, l\}$, and let $X_{l, m}$ be a cylinder process with intensity measure as in (5.1). Furthermore, we assume that there exist $L \in \operatorname{supp} \Phi$ and $K \in \operatorname{supp} \mathrm{P}(L, \cdot)$ such that $K$ is strictly convex in aff $K$. Then $X_{l, m}$ is weakly stationary and weakly isotropic if and only if $\mu_{z}$ is rotation invariant for all $z \in \mathbb{R}^{d}$.

Corollary 5.2. Let $d \geq 2, l \in\{1, \ldots, d\}$, and $m \in\{0, \ldots, l\}$, and let $X_{l, m}$ be a Poisson cylinder process with intensity measure as in (5.1). Furthermore, we assume that there exist $L \in \operatorname{supp} \Phi$ and $K \in \operatorname{supp} \mathrm{P}(L, \cdot)$ such that $K$ is strictly convex in aff $K$. Then $X_{l, m}$ is stationary and isotropic if and only if $\mu_{z}$ is rotation invariant for all $z \in \mathbb{R}^{d}$.

Remark. The question remains open of whether the additional assumption that there exist $L \in \operatorname{supp} \Phi$ and $K \in \operatorname{supp} \mathrm{P}(L, \cdot)$ such that $K$ is strictly convex in aff $K$ can be omitted in Theorem 5.2. The latter is directly related to the open problem if Theorem A.1 is true without the additional assumption that $K$ is strictly convex in aff $K$. It should also be added that the results of this paper do not depend on the choice of center function (in our case the Steiner point) that was used to obtain $\mathrm{P}_{0}$. Only the proof of Theorem A.1 has to be modified slightly as for any other choice of center function the origin no longer has to be a relative interior of $K$.

\section{Appendix A. Auxiliary results for convex bodies}

In this appendix we collect some results for convex bodies which are needed throughout the paper. Let $K \in \mathcal{K}$ be a convex body and $h(K, \cdot)$ its support function. The Steiner point $s(K)$ of $K$ is defined as

$$
s(K):=\frac{1}{\lambda_{d}\left(B^{d}\right)} \int_{S^{d-1}} h(K, u) \omega_{d-1}(\mathrm{~d} u) .
$$

For any $g \in G_{d}, s(g K)=g s(K)$ and $s(K)$ always lies in the relative interior of $K$. More details on the Steiner point can be found in [5, pp. 42-43]. Now additionally assume that $K$ has a nonempty interior. The map $\sigma_{K}: \operatorname{reg} K \rightarrow S^{d-1}$ is continuous; see [5, p. 78]). Furthermore, we provide a formula for integrals with respect to the $(d-1)$ th support measure, which follows directly from Lemma 2.1 of [9].

Lemma A.1. Let $K \in \mathcal{K}$ with nonempty interior and let $f: \mathbb{R}^{d} \times S^{d-1} \rightarrow[0, \infty)$ be measurable. Then

$$
\int_{\mathbb{R}^{d} \times S^{d-1}} f(x, u) \Xi_{d-1}(K, \mathrm{~d} x \times \mathrm{d} u)=\int_{\mathbb{R}^{d}} f\left(x, \sigma_{K}(x)\right) C_{d-1}(K, \mathrm{~d} x) .
$$

We will also need the following lemmas.

Lemma A.2. Let $f: \mathbb{R}^{d} \rightarrow[0, \infty)$ be continuous and $f \not \equiv 0$. Then, for all $n \in \mathbb{N}$, there exists an $r(n) \in[0, \infty)$ such that

$$
\int_{\mathbb{R}^{d}} \int_{r(n) B^{d}} f(z-x) \lambda_{d}(\mathrm{~d} z) \Xi_{d-1}\left(K, \mathrm{~d} x \times S^{d-1}\right)>0
$$

for all $K \in \mathcal{K}_{0}^{(n)}:=\left\{K \in \mathcal{K}_{0} \mid \operatorname{dim} K \in\{d-1, d\}, K \subseteq n B^{d}\right\}$. 
Proof. The set $T:=\left\{z \in \mathbb{R}^{d} \mid f(z)>0\right\} \neq \varnothing$. Hence, there exists an $r>0$ such that $T \cap r B^{d} \neq \varnothing$. We define $r(n):=r+n$ and let $x \in n B^{d}$. For $t \in\left(T \cap r B^{d}\right),(t+x) \in r(n) B^{d}$. This implies that

$$
\int_{r(n) B^{d}} f(z-x) \lambda_{d}(\mathrm{~d} z) \geq \int_{x+\left(T \cap r B^{d}\right)} f(z-x) \lambda_{d}(\mathrm{~d} z)=\int_{T \cap r B^{d}} f(z) \lambda_{d}(\mathrm{~d} z)>0 .
$$

Lemma A.3. Let $m \in\{0, \ldots, d\}, K \in \mathcal{K}_{0}$, and $\left(K_{i}\right)_{i \in \mathbb{N}} \subseteq \mathcal{K}_{0}$. Furthermore, we assume that $\operatorname{dim} K=\operatorname{dim} K_{i}=m$ for all $i \in \mathbb{N}$ and that $K_{i} \rightarrow K$. For $h: S^{d-1} \times \mathbb{R}^{d} \rightarrow[0, \infty)$ continuous, we then have

$$
\lim _{i \rightarrow \infty} \int_{\text {rel bd } K_{i}} h\left(\tilde{\sigma}_{K_{i}}(x), x\right) \mathscr{H}^{m-1}(\mathrm{~d} x)=\int_{\text {rel bd } K} h\left(\tilde{\sigma}_{K}(x), x\right) \mathscr{H}^{m-1}(\mathrm{~d} x) .
$$

Proof. If $m=0$ the assertion is true since in this case $K_{i}=K=\{0\}$ for all $i \in \mathbb{N}$. If $m=d$ the theorem follows from Lemma A.1 and the weak continuity of the mapping $K \mapsto \Xi_{d-1}(K, \cdot)$. Hence, we assume that $m \in\{1, \ldots, d-1\}$. If $K_{i} \subseteq$ aff $K$ for almost all $i \in \mathbb{N}$ the assertion follows from the case in which $m=d$. Otherwise, since $\operatorname{dim} K=\operatorname{dim} K_{i}=m$ for all $i \in \mathbb{N}$, there exists a rotation $\vartheta_{i} \in S O_{d}$ such that $\vartheta_{i} K_{i} \subseteq$ aff $K$. We choose $\vartheta_{i}$ in such a way that $d\left(\vartheta_{i} K_{i}, K\right)$ is minimal. Obviously, $\vartheta_{i} K_{i}$ converges to $K$ and we have

$$
\lim _{i \rightarrow \infty} \int_{\text {rel bd } \vartheta_{i} K_{i}} h\left(\tilde{\sigma}_{\vartheta_{i} K_{i}}(x), x\right) \mathscr{H}^{m-1}(\mathrm{~d} x)=\int_{\text {rel bd } K} h\left(\tilde{\sigma}_{K}(x), x\right) \mathscr{H}^{m-1}(\mathrm{~d} x) .
$$

The continuity of $h$ and a compactness argument then yield the assertion.

One of the major tools used to prove the results of this paper is the following theorem.

Theorem A.1. Let $d \geq 2$, let $K \in \mathcal{K}_{0}$ such that $\operatorname{dim} K=m>0$, and let $K$ be strictly convex in aff $K$. Furthermore, let $f: \mathbb{R}^{d} \rightarrow[0, \infty)$ be a continuous function. Then $f$ is constant if and only if there exists a function $\eta: S O_{d} \rightarrow[0, \infty)$ such that

$$
\int_{\text {rel bd } K} f(z-\vartheta x) g\left(\tilde{\sigma}_{K}(x)\right) \mathscr{H}^{m-1}(\mathrm{~d} x) \eta(\vartheta)=\int_{\text {rel bd } K} f(z-x) g\left(\tilde{\sigma}_{K}(x)\right) \mathscr{H}^{m-1}(\mathrm{~d} x)
$$

for all $z \in \mathbb{R}^{d}, \vartheta \in S O_{d}$, and continuous $g: S^{d-1} \rightarrow[0, \infty)$.

Proof. The only-if part is obvious, so let us assume that (A.1) holds. First, let $m=d$. Recall that $\sigma_{K}$ is continuous on reg $K$ and that $\mathscr{H}^{d-1}$ (bd $\left.K \backslash \operatorname{reg} K\right)=0$. Since $K$ is strictly convex, no two points on the boundary of $K$ have the same outer normal vector. Therefore, for any $x \in \operatorname{reg} K$, we have

for all $\varepsilon>0$ there exists a $\delta>0$ for all $y \in \operatorname{reg} K$ such that

$$
\left\|\sigma_{K}(x)-\sigma_{K}(y)\right\|<\delta \Longrightarrow\|x-y\|<\varepsilon .
$$

Here $\|\cdot\|$ denotes the Euclidean norm. We fix $z \in \mathbb{R}^{d}$ and $x_{0} \in \operatorname{reg} K$. Furthermore, we assume that there exists a $\vartheta \in S O_{d}$ such that

$$
f\left(z-\vartheta x_{0}\right) \eta(\vartheta)>f\left(z-x_{0}\right) \eta(\vartheta) .
$$


Since $f$ is continuous, there exists a $\varepsilon>0$ such that $f(z-\vartheta y) \eta(\vartheta)>f(z-y) \eta(\vartheta)$ for all $y \in \mathbb{R}^{d}$ with $\left\|y-x_{0}\right\|<\varepsilon$. We choose $\delta>0$ as in (A.2) and define a mapping $g: S^{d-1} \rightarrow[0, \infty)$ by

$$
g(u):=1-\frac{1}{\delta}\left\|u-\sigma_{K}\left(x_{0}\right)\right\|
$$

for all $u \in\left(\sigma_{K}\left(x_{0}\right)+\delta B^{d}\right) \cap S^{d-1}$ and $g(u):=0$ elsewhere. Note that $g$ is continuous. Let $S:=\left(\sigma_{K}\left(x_{0}\right)+\delta\left(\right.\right.$ int $\left.\left.B^{d}\right)\right) \cap S^{d-1}$ be the set of all unit normals in a $\delta$-ball around $\sigma_{K}\left(x_{0}\right)$. By the continuity of $\sigma_{K}$,

$$
\mathscr{H}^{d-1}\left(\operatorname{reg} K \cap \sigma_{K}^{-1}(S)\right)>0 .
$$

Additionally, we have

$$
f(z-\vartheta x) \eta(\vartheta) g\left(\sigma_{K}(x)\right) \geq f(z-x) \eta(\vartheta) g\left(\sigma_{K}(x)\right)
$$

for all $x \in$ bd $K$ and

$$
f(z-\vartheta x) \eta(\vartheta) g\left(\sigma_{K}(x)\right)>f(z-x) \eta(\vartheta) g\left(\sigma_{K}(x)\right)
$$

for all $x \in \operatorname{reg} K \cap \sigma_{K}^{-1}(S)$. This yields a contradiction to (A.1). Hence,

$$
f(z-\vartheta x) \eta(\vartheta)=f(z-x)
$$

for all $\vartheta \in S O_{d}$ and all $x \in \operatorname{reg} K$. Since $f$ is continuous and reg $K$ is a dense subset of bd $K$, this holds for all $x \in$ bd $K$. Now let $\vartheta \in S O_{d}$ have the property that there exists a $u \in S^{d-1}$ with $\vartheta u=u$. Then we can find an $x \in$ bd $K$ with $x=\alpha u$ for some $\alpha \in(0, \infty)$ and, thus, $\vartheta x=x$. It follows, from (A.3) and $f \not \equiv 0$, that $\eta_{\vartheta^{-1}}\left(\vartheta^{-1} K\right)=1$. Let $x \in$ bd $K$ be fixed. If $d \geq 3$, for any $y \in\|x\| S^{d-1}$, we can find $\vartheta \in S O_{d}$ with the property that $\vartheta y=x$ and $u \in S^{d-1}$ with $\vartheta u=u$. As we just proved, the latter implies that $\eta(\vartheta)=1$. This yields $f(z-y)=f(z-x)$ and, thus, $f$ is constant on $z+\|x\| S^{d-1}$. This implies that, for $z \in \mathbb{R}^{d}$ and $y \in\left(z+2\|x\| S^{d-1}\right)$, we have

$$
f(z)=f(y) .
$$

For $z^{\prime}, z^{\prime \prime} \in \mathbb{R}^{d}$ with $\left\|z^{\prime}-z^{\prime \prime}\right\| \leq 2\|x\|$,

$$
\left(z^{\prime}+\|x\| S^{d-1}\right) \cap\left(z^{\prime \prime}+\left\|x_{0}\right\| S^{d-1}\right) \neq \varnothing
$$

and so $f\left(z^{\prime}\right)=f\left(z^{\prime \prime}\right)$ must hold. This can only be true if $f$ is constant on the whole of $\mathbb{R}^{d}$.

Let $d=2$. For $u \in S^{1}$, let $x=x(u), x^{\prime}=x^{\prime}(u) \in \operatorname{bd} K$ be the points on the boundary of $K$ that lie on the half-line $\{\alpha u \mid \alpha \geq 0\}$ and $\{\alpha u \mid \alpha \leq 0\}$, respectively. Furthermore, we define

$$
S:=\left\{x(u)-x^{\prime}(u) \mid u \in S^{d-1}\right\} .
$$

Note that $S$ is a closed curve and the boundary of a star-shaped set with nonempty interior. From (A.3) we can deduce that, for each $z \in \mathbb{R}^{2}$, the function $f$ is constant on $z+2 S$ and the latter implies that $f$ is constant.

Now let $m<d$. In aff $K, K$ is strictly convex, so (A.2) holds for $K$ if we replace $\sigma_{K}$ by $\tilde{\sigma}_{K}$ and reg $K$ by the set of all points on rel bd $K$ that have a unique outer normal vector in aff $K$. The assertion then follows as in the case in which $m=d$. 
Let $X$ be a particle process with intensity measure as in (3.1). Throughout the paper we have implicitly used the fact that the measure

$$
\mathcal{A} \mapsto \int_{\mathcal{K}_{0}} \int_{\mathbb{R}^{d}} \mathbf{1}_{\mathcal{A}}(x, K) \Xi_{d-1}\left(K, \mathrm{~d} x \times S^{d-1}\right) \mathrm{P}_{0}(\mathrm{~d} K)
$$

on $\mathcal{B}\left(\mathbb{R}^{d}\right) \otimes \mathscr{B}\left(\mathcal{K}_{0}\right)$ is $\sigma$-finite. Define $\mathcal{K}_{0}^{(n)}:=\left\{K \in \mathcal{K}_{0} \mid K \subseteq n B^{d}\right\}$; it is easy to check that $\mathcal{A}_{n}:=\mathbb{R}^{d} \times \mathcal{K}_{0}^{(n)}$ has finite measure.

\section{Appendix B. Auxiliary results for cylinders}

In Section 5 we used the following lemmas.

Lemma B.1. The sequence $\left(K_{i}+L_{i}\right)_{i \in \mathbb{N}} \subseteq \mathcal{Z}_{q, 0}^{d}$ converges to $K+L \in \mathcal{Z}_{q, 0}^{d}$ in $\mathcal{F}^{\prime}$ if and only if $K_{i}$ converges to $K$ with respect to the Hausdorff metric and $L_{i}$ to $L$ in $\mathcal{F}^{\prime}$.

Proof. The only-if part holds by Theorem 1.2.3 of [8]. For the if part, note that $\mathcal{L}_{q}^{d}$ is compact and metrizable; see [7, Chapter 1]. Hence, any sequence $\left(L_{i}\right)_{i \in \mathbb{N}}$ of linear subspaces contains a convergent subsequence. Using Theorem 1.1.2 of [8] and the definition of the Hausdorff metric it is a straightforward calculation to show that the assertion holds.

Lemma B.2. Let $l \in\{0, \ldots, d\}$, let $z \in \mathbb{R}^{d}$, and let $f: \mathcal{E}_{d-l}^{d} \rightarrow[0, \infty)$ and $g: S^{d-1} \rightarrow$ $[0, \infty)$ be continuous functions. Then the mapping

$$
\mathbb{Z}_{d-l, 0}^{d} \rightarrow[0, \infty), K+L \mapsto \int_{\text {rel bd } K} g\left(\tilde{\sigma}_{K}(x)\right) f(L+z-x) \mathscr{H}^{m-1}(\mathrm{~d} x),
$$

is continuous.

Proof. Let $K+L \in \mathcal{Z}_{d-l, 0}^{d}$ be fixed and let $\left(K_{i}+L_{i}\right)$ be a sequence in $\mathcal{Z}_{d-l, 0}^{d}$ converging to $K+L$. By Lemma B.1 and a compactness argument, for all $\varepsilon>0$, there exists an $i_{0} \in \mathbb{N}$ with the property that

$$
\max _{x \in \text { rel bd } K_{i}}\left|f\left(L_{i_{n}}+z-x\right)-f(L+z-x)\right|<\varepsilon
$$

for all $i \geq i_{0}$. This implies that, for all $\varepsilon^{\prime}>0$, we can find an $i_{0}^{\prime} \in \mathbb{N}$ such that

$$
\begin{aligned}
& \int_{\text {rel bd } K_{i}} g\left(\tilde{\sigma}_{K_{i}}(x)\right) f(L+z-x) \mathscr{H}^{m-1}(\mathrm{~d} x)-\varepsilon^{\prime} \\
& \leq \int_{\text {rel bd } K_{i}} g\left(\tilde{\sigma}_{K_{i}}(x)\right) f\left(L_{i}+z-x\right) \mathscr{H}^{m-1}(\mathrm{~d} x) \\
& \leq \int_{\text {rel bd } K_{i}} g\left(\tilde{\sigma}_{K_{i}}(x)\right) f(L+z-x) \mathscr{H}^{m-1}(\mathrm{~d} x)+\varepsilon^{\prime}
\end{aligned}
$$

holds for all $i \geq i_{0}^{\prime}$. Combined with Lemma A.3 this yields the assertion.

\section{References}

[1] Fallert, H. (1996). Quermaßdichten für Punktprozesse konvexer Körper und Boolesche Modelle. Math. Nachr. 181, 165-184.

[2] Hoffmann, L. M. (2007). Intersection densities for nonstationary Poisson processes of hypersurfaces. Adv. Appl. Prob. 39, 307-317. 
[3] Hug, D. And Last, G. (2000). On support measures in Minkowski spaces and contact distributions in stochastic geometry. Ann. Prob. 28, 796-850.

[4] Matheron, G. (1975). Random Sets and Integral Geometry. John Wiley, New York.

[5] Schneider, R. (1993). Convex Bodies: The Brunn-Minkowski Theory. Cambridge University Press.

[6] Schneider, R. (2003). Nonstationary Poisson hyperplanes and their induced tessellations. Adv. Appl. Prob. 35, 139-158.

[7] SChNeIDER, R. AND WeIL, W. (1992). Integralgeometrie. Teubner, Stuttgart.

[8] SChNeIDER, R. AND WeIL, W. (2000). Stochastische Geometrie. Teubner, Stuttgart.

[9] WeIL, W. (1997). Mean bodies associated with random closed sets. Rend. Circ. Mat. Palermo (2) Suppl. 50, 387-412.

[10] WeIL, W. (1997). On the mean shape of particle processes. Adv. Appl. Prob. 29, 890-908. 